\title{
MeCP2 gates spatial learning-induced alternative splicing events in the mouse hippocampus
}

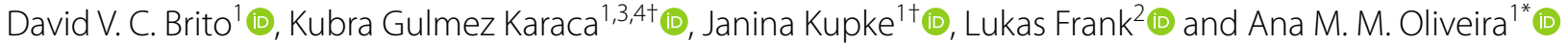

\begin{abstract}
Long-term memory formation is supported by functional and structural changes of neuronal networks, which rely on de novo gene transcription and protein synthesis. The modulation of the neuronal transcriptome in response to learning depends on transcriptional and post-transcriptional mechanisms. DNA methylation writers and readers regulate the activity-dependent genomic program required for memory consolidation. The most abundant DNA methylation reader, the Methyl $\mathrm{CpG}$ binding domain protein 2 (MeCP2), has been shown to regulate alternative splicing, but whether it establishes splicing events important for memory consolidation has not been investigated. In this study, we identified the alternative splicing profile of the mouse hippocampus in basal conditions and after a spatial learning experience, and investigated the requirement of $\mathrm{MeCP} 2$ for these processes. We observed that spatial learning triggers a wide-range of alternative splicing events in transcripts associated with structural and functional remodeling and that virus-mediated knockdown of MeCP2 impairs learning-dependent post-transcriptional responses of mature hippocampal neurons. Furthermore, we found that MeCP2 preferentially affected the splicing modalities intron retention and exon skipping and guided the alternative splicing of distinct set of genes in baseline conditions and after learning. Lastly, comparative analysis of the MeCP2-regulated transcriptome with the alternatively spliced mRNA pool, revealed that MeCP2 disruption alters the relative abundance of alternatively spliced isoforms without affecting the overall mRNA levels. Taken together, our findings reveal that adult hippocampal MeCP2 is required to finetune alternative splicing events in basal conditions, as well as in response to spatial learning. This study provides new insight into how MeCP2 regulates brain function, particularly cognitive abilities, and sheds light onto the pathophysiological mechanisms of Rett syndrome, that is characterized by intellectual disability and caused by mutations in the Mecp2 gene.
\end{abstract}

Keywords: Alternative splicing, Adult brain, DNA methylation, Gene transcription, Rett syndrome, RNA sequencing

\section{Introduction}

It is well established that long-term memory formation requires de novo gene transcription and protein synthesis. In response to neuronal activity, immediate early

\footnotetext{
*Correspondence: oliveira@nbio.uni-heidelberg.de

${ }^{\dagger}$ Kubra Gulmez Karaca and Janina Kupke equally contributed to this work ${ }^{1}$ Department of Neurobiology, Interdisciplinary Centre for Neurosciences (IZN), Heidelberg University, Im Neuenheimer Feld 366, 69120 Heidelberg, Germany
}

Full list of author information is available at the end of the article genes are rapidly transcribed, many of which initiate a second, delayed wave of gene transcription [1]. These newly synthesized mRNAs and proteins guide neuronal structural and functional changes that support memory consolidation [1, 2]. This complex process is regulated at multiple layers by the action of transcription factors and epigenetic players as well as chromatin architecture organizers that alter the accessibility of transcribed loci [3-7]. At the same time, neuronal activity triggers alternative splicing events that offer another level of 
gene expression regulation. Indeed, several studies have reported activity-dependent alternative splicing mechanisms in neurons [8-12] whose disruptions are associated with brain disorders [13]. Furthermore, selective expression of alternative splice variants functionally impacts cells through the remodeling of the transcriptome which may modify protein interaction, function and localization [10, 14-16]. Altogether, these findings strongly suggest that the coordinated regulation of gene transcription and alternative splicing is vital to determine neuronal activity-dependent changes required for memory consolidation.

DNA methylation is a dynamically regulated epigenetic mark that controls activity-dependent transcription and alternative splicing $[5,17]$. Methyl CpG binding domain protein 2 (MeCP2) is the most abundant DNA methylation reader in the brain, linking DNA methyl marks to higher order chromatin architectural changes through interaction with its numerous binding partners $[18,19]$. $\mathrm{MeCP} 2$ function is essential during neurodevelopment, since reduction in MeCP2 levels culminates in a severe neurological disorder, Rett Syndrome (RTT) [20]. Similarly, MeCP2 is indispensable during adulthood; it gates adult cognitive abilities and maintains chromatin architecture and proper functioning of the brain $[18,21]$. Until now, MeCP2 has been repeatedly shown to impact the transcriptional profile of developing and mature neurons in basal conditions [22], as well as in response to neuronal activity [23, 24]. In contrast, less is known about its functions in alternative splicing mechanisms regulating synaptic plasticity changes required for the formation and maintenance of memory. Recent studies identified that MeCP2 interacts with alternative splicing components, (e.g., Y-box binding protein 1 [YB-1]), and regulates their expression to influence alternative splicing events in neuroblastoma [25] or cancer cell lines [26]. Reduced DNA methylation that leads to reduced binding of MeCP2 to DNA was shown to decrease alternative splicing events and increased intron-retention mechanisms in embryonic stem cells, and in human and mouse cell lines [27]. Moreover, in mouse models of RTT, MeCP2 was shown to control alternative splicing events in the cortex during basal conditions $[25,28]$ and in the hippocampus in basal state and in a seizure model [8]. Altogether, these studies have attributed a role for MeCP2 in the regulation of alternative splicing. However, it remains unclear whether $\mathrm{MeCP} 2$ establishes alternative splicing events important for memory consolidation in response to a physiological learning stimulus.

Therefore, in this study we aimed to investigate the alternative splicing regulatory function of MeCP2 in the adult hippocampus of mice during spatial memory consolidation. We used a previously characterized mouse model in which MeCP2 is selectively reduced in the adult hippocampus to exclude possible confounds originating from impaired development or from abnormal anxiety and motor abilities [21]. Using this model, we performed RNA-sequencing (RNA-seq) to determine genome-wide alternative splicing events regulated by $\mathrm{MeCP} 2$ in basal conditions, as well as after a non-aversive spatial learning task. We identified a novel set of learning-induced alternative splicing variants in the mouse hippocampus. Furthermore, we found that MeCP2 knockdown altered the alternatively spliced mRNA profile of hippocampal neurons in basal conditions and abolished the alternative splicing events triggered by learning, mostly affecting exon-exclusion and intron-retention mechanisms involved in synaptic plasticity. Moreover, by comparative analysis of MeCP2-regulated transcriptome with alternatively spliced mRNA pool, we provided evidence that MeCP2 knockdown altered the relative abundance of alternatively spliced mRNAs even if the overall levels of the gene were not changed. Overall, our results attribute a novel role to MeCP2 in guiding basal and learning-induced alternative splicing mechanisms in mature hippocampal neurons required for long-term memory formation.

\section{Materials and methods \\ Mice}

Throughout the study, we used adult male C57BL/6N mice that were 8 weeks old at the time of surgery [(MeCP2-shRNA $(\mathrm{n}=8)$ or Control-shRNA $(\mathrm{n}=8)]$ (Charles River, Sulzfeld, Germany). The mice were grouphoused on a $12 \mathrm{~h}$ light/dark cycle with ad libitum access to food and water. All behavioral experiments were carried out during the light phase. Mice that were sick and/ or injured from cage-mate fighting were not included into the study. All procedures were performed according to the German guidelines for the care and use of laboratory animals and with the European Community Council Directive 86/609/EEC.

\section{Recombinant adeno-associated virus (rAAV)}

Viral particles were produced and purified as described previously [29]. For the knockdown of MeCP2, we used a vector containing the $\mathrm{U} 6$ promoter upstream of the short-hairpin RNA (shRNAs) (MeCP2-specific or control) sequence and a CamKII $\alpha$ promoter driving mCherry expression (as an infection control) as described previously [21].

\section{Stereotaxic surgery}

A total volume of $1.5 \mu \mathrm{l}(2: 1$ mixture of rAAV solution and $20 \%$ mannitol) was injected into the dorsal hippocampus (relative to Bregma: $-2 \mathrm{~mm}$ anteroposterior, $\pm 1.5 \mathrm{~mm}$ 
mediolateral, $-1.7,-1.9$ and $-2.1 \mathrm{~mm}$ dorsoventral) at the speed of $200 \mathrm{nl} / \mathrm{min}$ as previously described [21, 30].

\section{Spatial-object recognition task}

Spatial-object recognition task was executed as described previously [31]. Briefly, after mice were habituated to the experimenter and behavioral room by gentle handling ( 3 consecutive days, 1 min per mouse), mice were placed into an open arena $(50 \mathrm{~cm} \times 50 \mathrm{~cm} \times 50 \mathrm{~cm}$ with a visual cue placed on the arena wall) in the absence of objects. In the next three sessions, mice were able to explore two distinct objects (a glass bottle and a metal tower). Between the sessions, mice were placed in their home cage for $3 \mathrm{~min}$.

\section{RNA-sequencing}

30 min after training in spatial object recognition task, the infected dorsal hippocampal tissue (identified by mCherry expression) was micro-dissected for RNA-seq analysis. Home-cage mice were not subjected to training, but dissected simultaneously with trained mice to account for time of the day differences. Total RNA was isolated using the RNeasy Plus Mini Kit (Qiagen, Hilden, Germany) with additional on-column DNase I digestion according to the manufacturer's instructions and $1 \mu \mathrm{g}$ of total RNA from each sample was used for RNA-seq. Both differential gene expression (DEG) and differential alternative splicing (DAS) expression analysis was performed by GATC Biotech (Inview Transcriptome Discover, GATC Biotech AG, Constance, Germany) as previously described [21, 32]. In brief, paired-end sequencing libraries prepared from total RNA (see above) were sequenced on an Illumina HiSeq4000 platform. After removal of poor quality and single reads without mates, reads were aligned to the GRCm $38 / \mathrm{mm} 10$ mouse genome assembly with TopHat/Bowtie [33] guided by the Ensembl v85 transcript annotation, yielding between $\sim 73$ and 101 million mapped reads per sample. Differential gene expression (DEG) analysis was conducted using Cufflinks/ Cuffdiff [34] which computes per gene FPKM values to then test for differential expression between conditions. Differential alternative splicing (DAS) analysis was conducted using multivariate analysis of transcript splicing (MATS) [32]. TopHat/Bowtie aligned reads were used as input for MATS which compares splicing patterns by considering exon-exon junction read counts for transcript variants of a gene. For differential alternative splicing and statistical testing, MATS relies on a multivariate uniform prior to model the between-sample correlation in exon splicing patterns, and a Markov chain Monte Carlo method coupled with a simulation-based adaptive sampling procedure to calculate the p-values and false discovery rates (FDR). A $P_{\text {adjusted }}<0.05$ (FDR adjusted P-value) was used as a cut-off for DAS events. DASs above the cutoff were analyzed for enrichment of gene ontology (GO) terms and pathways using database for annotation, visualization and integrated discovery (DAVID) v6.8 (Huang da, Sherman, \& Lempicki, 2009a, 2009b). Default settings of DAVID were chosen except that the background database was restricted to the pool of genes annotated in our RNA-seq analysis [21]. Only gene enriched terms with a $-\log _{10} \mathrm{P}$-value $<3$ $\left(\mathrm{p}_{\text {value }}<0.001\right)$ were considered significant. Delta "percent spliced in" ( $\triangle \mathrm{PSI})$ distribution for two groups considered only DAS events detectable in both conditions tested as DAS events detected only in one of the comparisons were not categorized as unique or common in Figs. 2e and 4e. The DEGs identified in our previous study [21] were used for overlap analysis between DEGs and DAS in Figs. 3c, $d$ and $5 \mathrm{c}, \mathrm{d}$.

\section{Primer design}

Splice variant specific qRT-PCR and RT-PCR primers were designed with Primer3 (https://primer3.ut.ee) using the RefSeq curated (if available) or the GENCODE VM23 comprehensive transcript annotation and the GRCm38/ mm10 mouse genome assembly. Primer specificity and amplicon product sizes were confirmed by BLAST Search and in silico PCR (UCSC Genome Browser, mm10). Primer pair efficiencies and product melting curves were experimentally validated by qRT-PCR on serially diluted cDNA from primary mouse hippocampal cultures (see section qRT-PCR). All primers used in this study are listed in Additional file 1: Table S1.

\section{qRT-PCR}

RNA samples generated in our previous study [21] were used for qRT-PCR validation. These included the samples analyzed by RNA-seq and independent biological replicates. Total RNA was isolated from hippocampal tissue using the RNeasy Plus Mini Kit (Qiagen) with additional on-column DNase I digestion, according to the manufacturer's instructions. cDNA was synthesized from 400 ng RNA using the Applied Biosystems High Capacity Complementary DNA Reverse Transcription Kit (ThermoFisher Scientific). qRT-PCR was performed with the Power SYBR Green PCR Master Mix (Applied Biosystems) on a StepOnePlus Real-Time PCR System (Applied Biosystems). PCR reactions were run as technical triplicates in $10 \mu \mathrm{L}$ reactions (96-well format) with a final primer concentration of $0.5 \mu \mathrm{M}$ (each). $2 \mu \mathrm{L}$ of diluted cDNA $(\sim 1.25 \mathrm{ng}))$ were used per reaction. The following settings were used for thermo-cycling: $10 \min 95{ }^{\circ} \mathrm{C}, 40$ cycles of $10 \mathrm{~s}$ each $95^{\circ} \mathrm{C}, 60^{\circ} \mathrm{C}, 72^{\circ} \mathrm{C}$ followed by $15 \mathrm{~s}$ incubation at $95^{\circ} \mathrm{C}$. Melt curves were determined by subsequent heating from $60^{\circ} \mathrm{C}$ to $90{ }^{\circ} \mathrm{C}$ with a ramp speed of 
$0.6^{\circ} \mathrm{C} / \mathrm{min}$. Relative expression levels of each target transcript were determined by the $\Delta \Delta \mathrm{Ct}$ method using betaactin mRNA levels as reference.

\section{RT-PCR}

PCR reactions were performed using Q5 High-Fidelity Polymerase (NEB) according to the manufacturer's instructions with a final primer concentration of $0.5 \mu \mathrm{M}$ (each) and $1.25 \mathrm{ng}$ of diluted cDNA. The following settings were used for thermo-cycling: $30 \mathrm{~s} 98^{\circ} \mathrm{C}, 30$ cycles of $15 \mathrm{~s}$ of $98{ }^{\circ} \mathrm{C}, 20 \mathrm{~s}$ of $60^{\circ} \mathrm{C}, 20 \mathrm{~s}$ of $72^{\circ} \mathrm{C}$ followed by $2 \mathrm{~min}$ at $72{ }^{\circ} \mathrm{C}$. PCR products were separated on $2 \%$ agarose gels and visualized by Midori Green Xtra (Nippon Genetics).

\section{Data and statistical analysis}

Each set of experiments contained mice injected with control or experimental viruses and were randomized per cage (i.e., each cage of four mice contained mice injected with control or experimental viruses). After stereotaxic surgery and until the end of each experiment, the experimenter was blind to the identity of the virus injected into each mouse. Behavioral experiments were performed three weeks after stereotaxic delivery of rAAVs. Gene enrichment analysis was performed using Fisher's exact test $\mathrm{P}<0.001$. Cumulative analysis was performed using paired two-tailed Student's t test or Wilcoxon test, for normally and non-normal distributed data, respectively, p-values are shown on top of each panel. qRT-PCR data was analyzed using unpaired two-tailed Student's t test or Mann-Whitney test for normally and non-normally distributed data, respectively. Statistics were performed using GraphPad prism for Mac OS X, version 8.

\section{Gene expression omnibus (GEO) accession}

The RNA-seq data for alternative splicing analyzed in this study is publicly available at the National Center for Biotechnology Information (NCBI) Gene Expression Omnibus with the accession number GSE107004.

\section{Results}

Spatial learning induces alternative splicing events that are altered in MeCP2 knockdown mice

In this study, we sought to investigate whether MeCP2 regulates alternative splicing events in the adult hippocampus in basal conditions as well as after spatial learning. To this end, we delivered recombinant adenoassociated viruses (rAAV) into the adult dorsal hippocampus encoding either a control (Control-) or a MeCP2-specific shRNA sequence (Fig. 1). We knocked down $\mathrm{MeCP} 2$ in neurons by using an AAV serotype (rAAV1/2) that displays predominant neuronal tropism $[35,36]$. The viral construct also contained an expression cassette for mCherry under the control of the CamKII promoter (Fig. 1) that served as an infection marker. This strategy allowed us to investigate $\mathrm{MeCP} 2$ function in the adult hippocampus without confounds originating from impaired postnatal neurodevelopment. We previously confirmed that this tool significantly decreases MeCP2 mRNA and protein levels selectively in the hippocampus. Moreover, MeCP2-shRNA mice displayed impairments in hippocampus-dependent long-term memory without

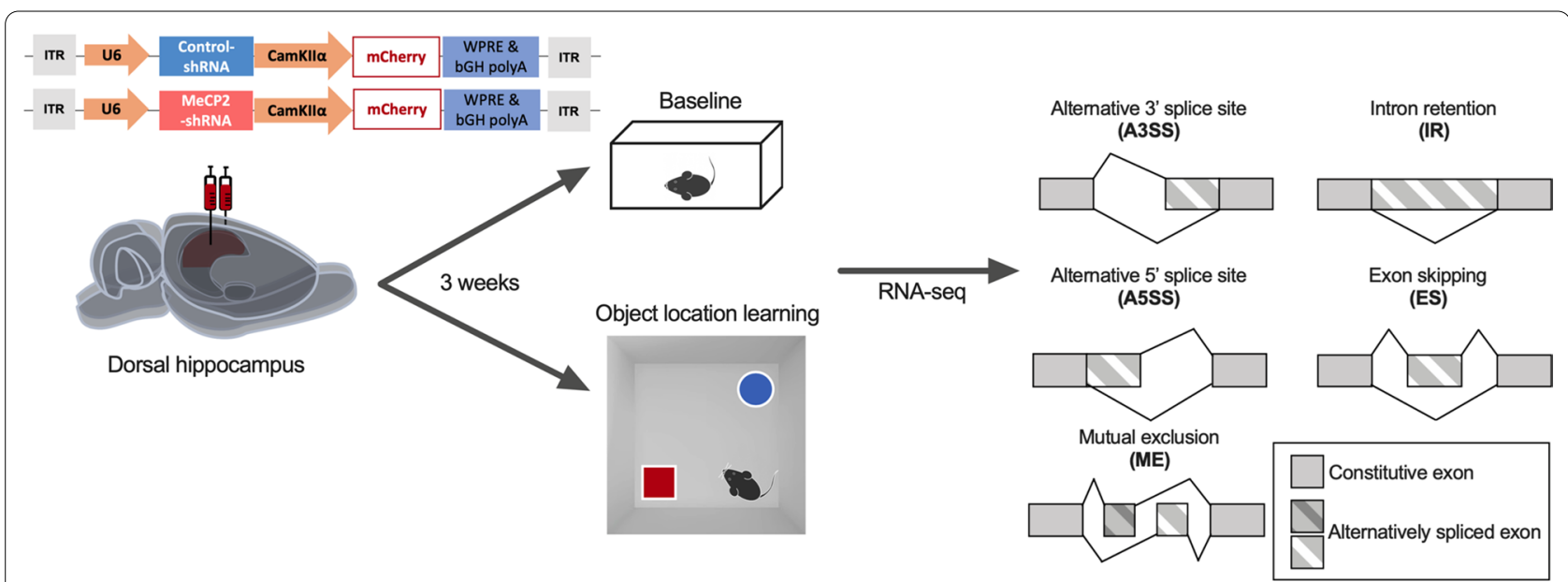

Fig. 1 Schematic representation of the experimental design and alternative splicing events analyzed in this study. The viral vector contains a U6 promoter driving expression of MeCP2-shRNA or a control-shRNA and mCherry expression under the CamKlla promoter. Three weeks after the delivery of recombinant AAVs into the dorsal hippocampus, mice remained either in home-cage (Baseline) or trained in object location learning. 30 min after training mice dorsal hippocampi were micro dissected and RNA-seq and alternative splicing analysis was performed. bGH polyA bovine growth hormone polyadenylation signal, ITR inverted terminal repeat, WPRE Woodchuck Hepatitis virus post-transcriptional regulatory element 
exhibiting broad neurological impairments, such as motor deficits or anxiety-like behavior [21] that typically occur in animal models with disrupted MeCP2 expression from early developmental stages. Thus, this experimental strategy was chosen for gene expression analysis. In this experiment, half of the mice were kept in their homecage (baseline), whereas the remaining underwent spatial object location training (learning) (Fig. 1). This behavioral protocol allowed us to detect hippocampal transcriptional changes associated with novel environment exposure and learning the location of objects in space. $30 \mathrm{~min}$ after the end of the task, a time point with robust transcriptomic changes after learning [21], we performed genome-wide differential alternative splicing analysis of the mouse dorsal hippocampal tissue expressing either MeCP2-shRNA or Control-shRNA in baseline conditions and after learning. RNA-seq analysis allowed the identification of five distinct mRNA splicing events: alternative $3^{\prime}$ splice sites (A3SS), alternative $5^{\prime}$ splice sites (A5SS), mutually exclusive exons (ME), intron retention (IR) and exon skipping (ES) events (Fig. 1).

We started by identifying the DAS events induced by spatial learning in control mice, and then asked whether $\mathrm{MeCP} 2$ knockdown alters this learning-induced alternative splicing program. To determine this, we compared the alternative splicing profile of each treatment condition (Control- or MeCP2-shRNA) in basal conditions versus after learning (Fig. 2a). We observed that object location learning induced 28 differential alternative splicing events in Control-shRNA-injected mice hippocampi, that consisted predominantly of ES events (42.8\%) followed by A5SS (21.4\%) and IR (17.9\%), A3SS (17.9\%) while no ME events were detected (Fig. 2b) [see Additional file 2: Table S2]. Some of the genes identified here have been previously described to undergo alternative splicing during memory consolidation or recall in a contextual fear conditioning paradigm (Dnajb5 and March7, Zfp207, Gls, Fuz, respectively) [9]. In contrast, in MeCP2-shRNA expressing hippocampi, 13 learningtriggered DAS events were detected (Fig. 2b). Furthermore, MeCP2-shRNA mice showed a clear shift towards more IR events (53.8\%) and a reduced occurrence of ES (23.1\%) and ME (7.7\%), A5SS (7.7\%) and A3SS (7.7\%) in response to learning compared to the controls (Fig. 2b) [see Additional file 2: Table S2]. These findings indicate that $\mathrm{MeCP} 2$ reduction impaired DAS events in the adult hippocampus in response to spatial learning. Next, we analyzed whether there is a change in the fraction of the included or excluded isoforms in Control- or MeCP2shRNA expressing mice using the delta "percent spliced in" $(\triangle \mathrm{PSI})$. The $\triangle \mathrm{PSI}$ represents the difference between the ratio of transcripts that retain or exclude an intron/ exon in two conditions. A $\triangle$ PSI value above or below $0 \%$ indicates an increased or reduced inclusion of alternative introns/exons, respectively. This parameter allows to investigate whether MeCP2 regulates the inclusion of introns/exons in alternatively spliced transcripts. Although MeCP2 reduction altered inclusion $(\triangle \mathrm{PSI}>0)$ and exclusion $(\triangle \mathrm{PSI}<0)$ events of each splicing subtype (Fig. 2c and Additional file 3: Figure S1A-D), we focused on IR and ES events shown in Fig. 2d since the majority of DAS belonged to these splicing categories, and MeCP2 induced an ES-IR switch. We found that MeCP2-shRNA animals showed a mild shift towards excluded IR events (14.3\% included vs. $85.7 \%$ excluded) compared to controls (20\% included vs. $80 \%$ excluded) and a decrease of ES (control: $16.7 \%$ included vs $83.3 \%$ excluded; MeCP2-shRNA: $33.3 \%$ included vs $66.7 \%$. excluded) (Fig. 2d) [see Additional file 4: Table S3]. Similarly, hippocampal knockdown of MeCP2 led to alterations on A3SS, A5SS and ME inclusion/exclusion events (Additional file 3: Figure S1A-D). The majority of splicing events occurred in the same direction, that is inclusion or exclusion, in both control and MeCP2-shRNA animals (Fig. 2e) [see Additional file 4: Table S3]. Nonetheless, we also detected a subset of alternative splicing events that occurred in opposite directions, meaning that they underwent increased inclusion in MeCP2shRNA mice and increased exclusion in Control-shRNA mice or vice versa (e.g. Gls, Osmr, Trmt1, Irf7) [see Additional file 4: Table S3]. Remarkably, only 2 of the 13 DAS events observed in MeCP2-shRNA mice overlapped with the DAS events detected in Control-shRNA mice. This indicates that in MeCP2 knockdown conditions DAS events that occur in control conditions were no longer present (e.g. Zmynd8, Nr3c1, P2rx6, Dnajb5) and new spliced isoforms were generated (11 unique DAS; e.g. Atl2, Fhl1, Marchf7) (Fig. 2e). None of these events (neither overlapping nor unique) showed a bias towards any particular splicing type (Additional file 3: Figure S1E-I). Statistical analysis of all the DAS events detected in Control- or MeCP2-shRNA mice in response to learning did not show a statistically significant difference between the groups (Fig. $2 \mathrm{f}$ and Additional file 3: Figure S1J-K). Importantly, we validated several of the identified AS events by qRT-PCR and RT-PCR analysis of samples used for RNA-seq analysis and additional independent biological replicates (Additional file 5: Figure S2).

To gain further insight into the functional categories of identified learning-induced DAS, we performed gene ontology (GO) analysis. For this, we carried out two separate analysis for inclusion and exclusion DAS events (Fig. 3a, b). We found that in control animals that underwent learning, GO terms associated with "Dendritic spine" and "Positive regulation of spine development" were predominantly enriched in the inclusion group 


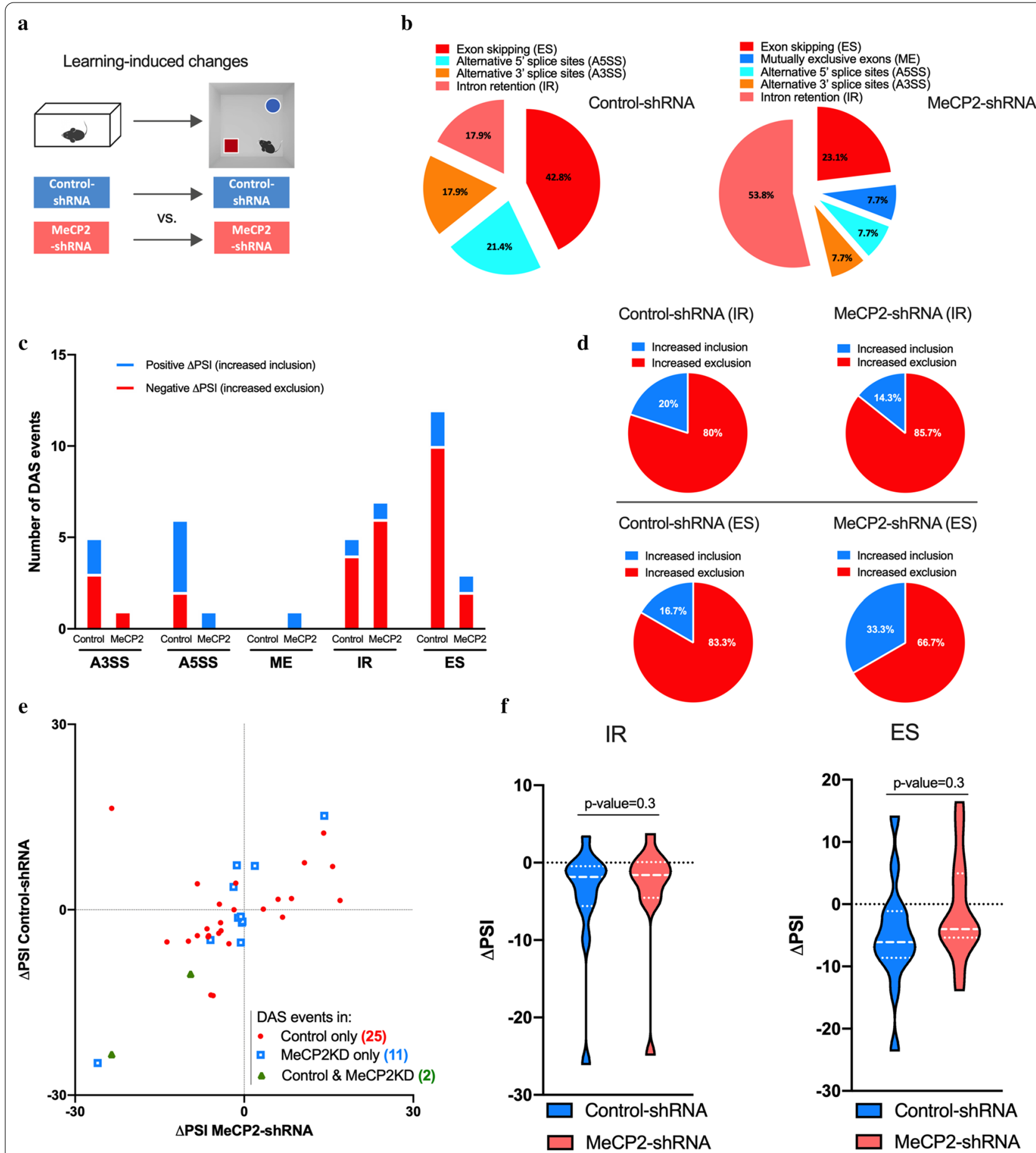

Fig. 2 Spatial learning induces alternative splicing events that are altered in MeCP2 knockdown mice. a Schematic representation of the comparisons used. b Proportion of each differential alternative splicing events (DAS) in control conditions (left) or in MeCP2 knock-down conditions (right) in learning-induced conditions. c Number of inclusion (positive $\triangle \mathrm{PSI}$, blue) and exclusion (negative $\triangle \mathrm{PSI}$, red) events for each type of alternative splicing modality in Control-shRNA (Control) and MeCP2-shRNA (MeCP2) mice (q-value < 0.05). d Pie charts showing the proportion of inclusion and exclusion events for intron retention (IR) and exon skipping (ES) in Control-shRNA and MeCP2-shRNA mice. e Scatter plots showing changes in IR and ES events in Control-shRNA (Control) and MeCP2-shRNA (MeCP2KD) mice upon learning. Red dots and blue squares represent alternative splicing events that occurred in either Control or MeCP2 knockdown hippocampi (q-value $<0.05$ ), respectively. Green triangles represent alternative splicing events that occurred in both conditions (q-value $<0.05$ ). $\mathbf{f}$ Violin plots showing the $\triangle P S I$ distribution of IR (left) and ES (right) events in Control-shRNA and MeCP2-shRNA hippocampi after learning. The P-values are based on paired two-tailed Student's t test or Wilcoxon test and are indicated at the top of each panel. $\triangle$ PSI: delta "percent spliced in" 


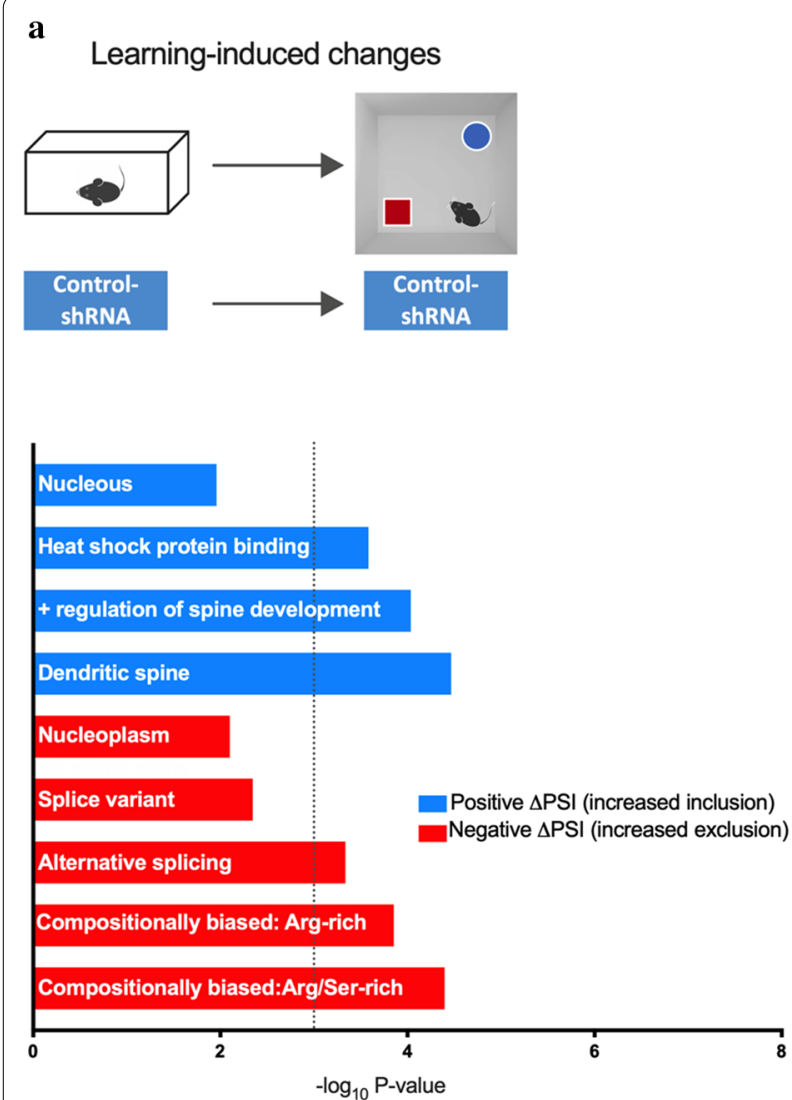

c Control-shRNA

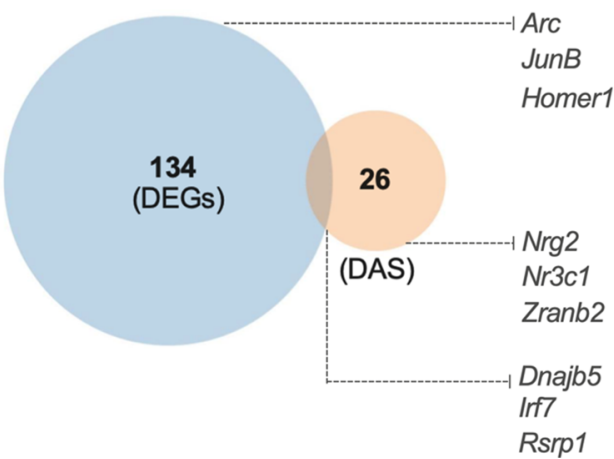

b
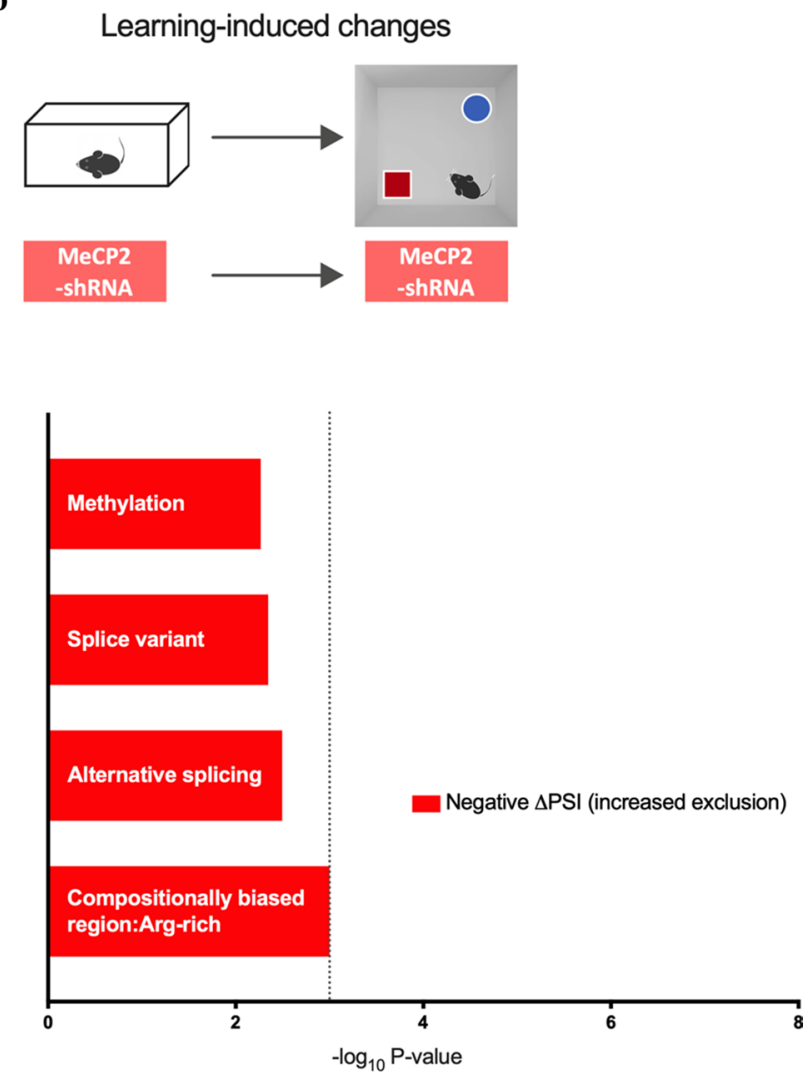

d

MeCP2-shRNA

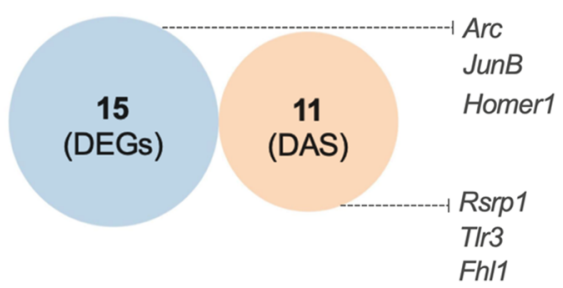

Fig. 3 Analysis of genes that underwent differential alternative splicing events upon spatial learning. $\mathbf{a}$, $\mathbf{b}$ Schematic representation of comparisons used (top). Gene ontology (GO) analysis for genes that underwent differential alternative splicing in the dorsal hippocampi of Control-shRNA (a) and MeCP2-shRNA (b) mice upon learning. Enriched GO terms (Fisher's exact test $\mathrm{P}<0.001$ ) for genes that underwent inclusion or exclusion ( $q$-value $<0.05$ ) events, upon learning. The blue and red bars represent - $\log _{10}(P$-value) of the GO enrichment for inclusion and exclusion events, respectively. The vertical dashed line serves as a marker for P-value $=0.001\left[-\log _{10}(P\right.$-value $\left.)=3\right]$. Absence of a colored bar means that genes of that GO term were not enriched in that specific category. $\mathbf{c}$ Venn diagram showing overlap between total number of differentially expressed genes (DEGs) and genes that underwent differential alternative splicing events (DAS) in home-cage (baseline) conditions when MeCP2 was knocked down in the adult dorsal hippocampus. $\mathbf{d}$ Venn diagram showing overlap between total number of differentially expressed genes and genes that underwent differential alternative splicing events in learning state (learning) conditions when MeCP2 was knocked down in the adult dorsal hippocampus 
$\left(-\log _{10} \mathrm{P}\right.$ value $\left.<3\right)$, whereas terms associated with "Alternative splicing" and "Splice variant" showed a trend for enrichment in the exclusion cohort $\left(-\log _{10} \mathrm{P}\right.$ value $\left.<3\right)$ [see Additional file 6: Table S4]. These findings suggest that learning-induced alternative splicing events in the hippocampi of control mice are associated with dendritic spine regulation. Notably, in MeCP2-shRNA mice, there was no enrichment detected for the inclusion group, and the exclusion DAS cohort showed a non-significant trend for enrichment for terms associated with "Methylation", "Splice variant", "Alternative splicing" and "Compositionally bias region: Arg/Ser-rich" [see Additional file 6: Table S4]. This data suggests that MeCP2 reduction compromises predominantly the learning-triggered processes associated with dendritic function.

Considering that MeCP2 is required for optimal expression and alternative splicing of several genes, we asked to which degree these two gene populations (DEGs and DASs) overlap. This analysis indicates whether $\mathrm{MeCP} 2$ uses these two regulatory mechanisms on similar or different genes. Since the same tissue was used for DAS and for the previously published differential gene expression analysis [21], the two datasets could be directly compared. To this end, we identified genes that underwent alternative splicing, and compared this gene population to the previously identified DEGs in the same conditions [21] (learning-induced DEGs were compared to learning-induced DASs in Control- or MeCP2-shRNA expressing mouse hippocampus) (Fig. 3c, d). We found that in control group, only 3 DEGs showed also DAS events in response to learning (out of 134 DEGs and 26 DAS) (Fig. 3c and Additional file 6: Table S4). In MeCP2shRNA animals, the differentially expressed genes in response to learning and the learning-induced differential alternative splicing events did not overlap (Fig. 3d and Additional file 6: Table S4). Taken together, this data indicates that learning induces changes in the expression levels and in the predominance of specific alternatively spliced variants of distinct gene populations. Furthermore, our results implicate a requirement for MeCP2 in both processes.

\section{MeCP2 knockdown changes splicing events in baseline and learning states}

To gain a deeper understanding of how MeCP2 regulates alternative splicing events, we asked whether acute MeCP2 reduction influences DAS events already in baseline and/or after learning conditions. To this end, we compared the alternative splicing profile of Control- versus MeCP2-shRNA mice in basal conditions, as well as after learning (hereafter, learning state) (Fig. 4a). We identified a total of 156 DAS events (q-value $<0.05$ ) in baseline conditions upon MeCP2 disruption in the hippocampus [see Additional file 7: Table S5]; ES events were predominant $(75 \%)$, followed by IR (10.3\%), ME (6.4\%), A5SS (4.5\%) and A3SS (3.8\%) (Fig. 4b). Altered alternative splicing in overlapping genes has been observed in the hippocampus of Mecp2-null mice (i.e. Zfp207, Map4 and Ppfia2) [8]. Similarly, the DAS profile of MeCP2-shRNA hippocampus after learning was different from the controls. We identified 94 DAS events (q-value $<0.05$ ) in MeCP2-shRNA mice in learning state [see Additional file 7: Table S5], in which ES events were predominant (70.2\%), followed by IR (25.5\%) and A3SS (4.3\%) whereas no A5SS and ME events were detected (Fig. 4b). Next, we determined the change in the fraction of the included or excluded events of each splicing subtype in baseline or learning in MeCP2-knockdown conditions (Fig. 4c, d). We found that MeCP2-shRNA animals have preferentially decreased IR in baseline conditions (31.2\% inclusion vs. $68.8 \%$ exclusion) while in learning state, the relative abundance of inclusions/exclusions in MeCP2-shRNA mice shifted predominantly towards included introns (66.7\% inclusion vs. $33.3 \%$ exclusion) (Fig. 4d) [see Additional file 8: Table S6]. Interestingly, although the total number of ES events in MeCP2-disrupted hippocampus decreased by learning (Fig. 4c), the proportion of inclusions/exclusions among the total ES events remained similar in baseline and learning state (baseline: $32.5 \%$ included vs. $67.5 \%$ excluded; learning 37.9\%: vs. 62.1\%) (Fig. 4d). Similarly, hippocampal knockdown of MeCP2 led to alterations on A3SS, A5SS and $\mathrm{ME}$ inclusion/exclusion events in baseline and in learning conditions (Additional file 9: Figure S3A-D) [see Additional file 8: Table S6]. Next, we checked the common DAS events in baseline and learning state in MeCP2-shRNA conditions. We found that hippocampal MeCP2 reduction led to 131 and 75 unique DAS events, in baseline (e.g. Gabrg2, Synj1, Map4) and in learning state (e.g. U2af1l4, Nrcam, P2xrx6, Gria3), respectively (Fig. 4e, Additional file 9: Figure S3). Only 19 DAS events occurred in both conditions, suggesting that learning induces distinct alternative splicing events. Notably, the majority of DAS events detected in baseline or after learning happened in the same direction in MeCP2-disrupted and control hippocampi, although a small subset of splicing events occurred in opposite ways (Fig. 4e and Additional file 8: Table S6). Deeper analysis revealed that the oppositely regulated DAS subset showed no bias for a particular splicing event type (Additional file 9: Figure S3E-I) [see Additional file 8: Table S6]. Cumulative analysis of all DAS events in MeCP2-disrupted hippocampus showed a significant increase in retained introns (Fig. 4f), a decrease in skipped exons (Fig. 4f) and either a significant or a trend for increase of A5SS and ME inclusion events in learning state compared to baseline (Additional 


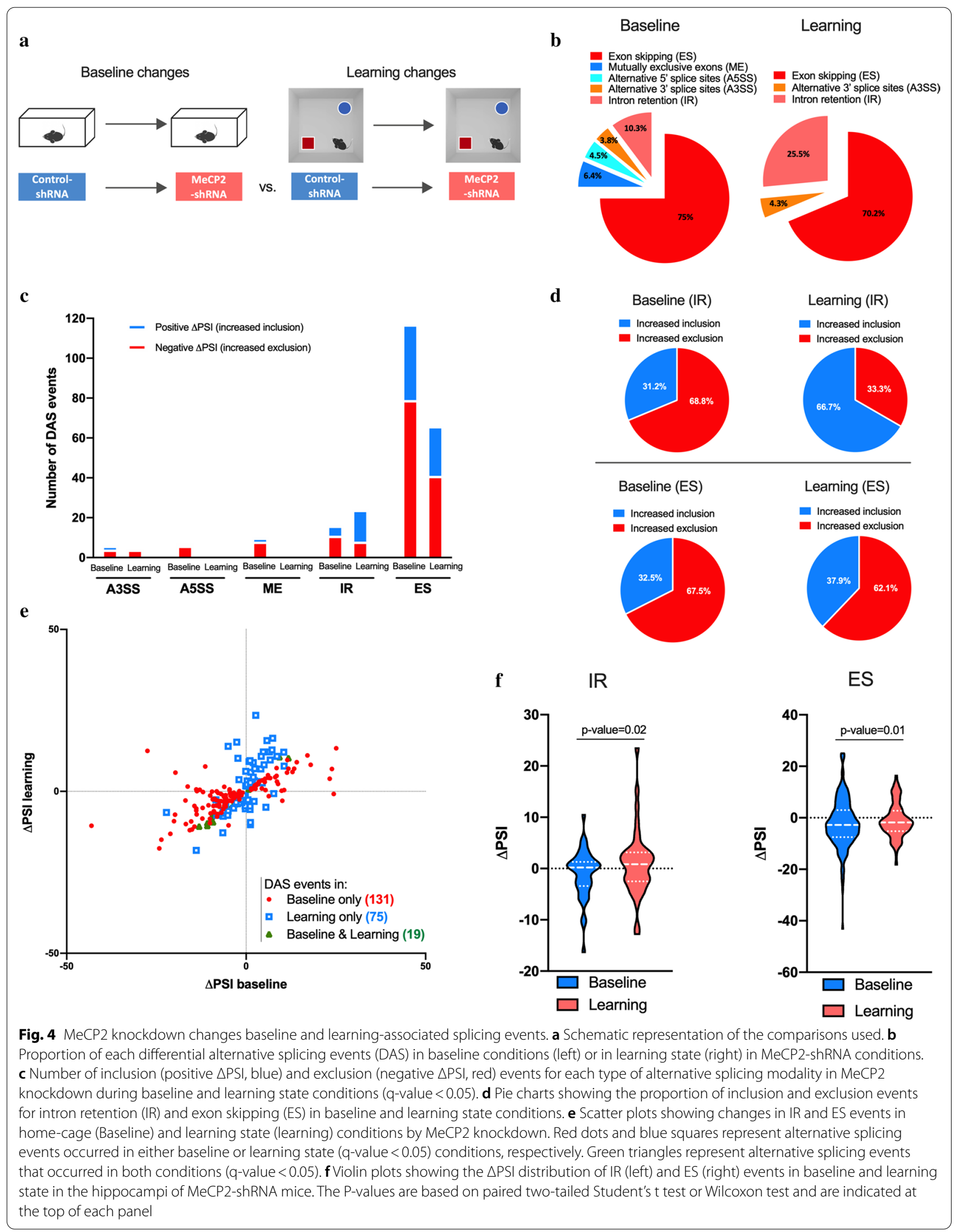


file 9: Figure S3J-L). No change was detected for A3SS events (Additional file 9: Figure S3J). These results show that $\mathrm{MeCP} 2$ reduction induces a distinct DAS profile in baseline and upon learning. Thus, indicating that the differences found in the learning state do not only reflect changes in basal conditions, but also a requirement for $\mathrm{MeCP} 2$ in learning-dependent alternative splicing. Importantly, we validated several of the identified AS events by qRT-PCR and RT-PCR analysis of samples used for RNA-seq analysis and additional independent biological replicates (Additional file 5: Figure S2).

Next, to gain insight into the functional categories of the genes that required $\mathrm{MeCP} 2$ for alternative splicing in baseline or learning states, we performed GO analysis. This was applied to both conditions (baseline or learning) and were divided into inclusion $(\triangle \mathrm{PSI}>0)$ and exclusion $(\triangle \mathrm{PSI}<0)$ events. We found that DAS inclusions in $\mathrm{MeCP} 2$ reduction in baseline conditions were enriched for terms such as "Phosphoprotein", "Alternative splicing" and "Cytoskeleton", whereas DAS exclusions in MeCP2shRNA mice were associated with the functional categories termed "Alternative splicing", "Clathrin vesicle coat", "Tubulin binding" $\left(-\log _{10} \mathrm{P}\right.$ value $<3$ ) (Fig. 5a) [see Additional file 10: Table S7]. After learning, only enrichment for "Alternative Splicing" for inclusion events and "Cellcell adherent junctions", "Neuronal cellular homeostasis" and "Positive regulation of protein binding" for increased exclusion events were found (Fig. 5b) [see Additional file 10: Table S7]. These results indicate that both in baseline and after learning conditions MeCP2 regulates DAS events associated with general neuronal function processes despite that DAS events are generally distinct in both conditions (Fig. 4e, f).

Next, we compared DEGs and DASs in MeCP2reduced hippocampi in baseline or learning states. We found that only 17 differentially expressed genes in MeCP2 knockdown also showed changed alternative splicing (out of 1948 DEGs and 130 DAS) in baseline conditions (Fig. 5c and Additional file 10: Table S7), whereas this number was as low as 7 genes in learning state (out of 884 DEGs and 82 DAS) (Fig. $5 \mathrm{~d}$ and Additional file 10: Table S7). Altogether, these findings indicate that MeCP2 regulates the predominance of specific alternatively spliced variants mostly without affecting the overall level of transcripts coded by that gene both in baseline conditions and after learning.

\section{Discussion}

In this study, we showed that adult hippocampal MeCP2 is required for the regulation of alternative splicing events during memory consolidation. We demonstrated that MeCP2 preserves the alternative splicing profile of mature hippocampal neurons and regulates learning-dependent splicing of genes important for neuronal structure and function. Therefore, our findings show that MeCP2 not only regulates the levels of expression of memory-related genes, but also the relative abundance of specific transcript isoforms, thus uncovering another mechanism by which MeCP2 impacts neuronal functional and structural properties during memory consolidation. This highlights a multifactorial requirement for $\mathrm{MeCP} 2$ in adult cognitive processes.

MeCP2 has well-established functions during neurodevelopment as evidenced by the severe neurological impairments characteristic of RTT, a neurodevelopmental disorder caused by mutations in the Mecp 2 gene [18, $20,37]$. Furthermore, several lines of evidence also support an important function during adulthood; MeCP2 is expressed at high levels in the adult brain [38] and is required for its function [21, 38-43]. Specifically, it has been demonstrated that $\mathrm{MeCP} 2$ plays an important role in adult cognitive abilities $[18,21]$. Mounting evidence indicates that long-lasting synaptic remodeling important for memory consolidation is supported not only by learning-triggered changes in the transcriptional, but also in the post-transcriptional profile [9] of neurons. In this study, we investigated the regulatory function of MeCP2 in alternative splicing mechanisms. To this end, we selectively decreased MeCP2 levels in adult hippocampal neurons [21]. This way, we could dissect the impact of $\mathrm{MeCP} 2$ disruption on the alternative splicing profile of mature hippocampal neurons without confounds originating from impaired neurodevelopment. We found that reducing MeCP2 expression of mature hippocampal neurons led to aberrant alternative splicing profiles. This finding is in line with previous studies that demonstrated a role for $\mathrm{MeCP} 2$ in alternative splicing regulation [25, $27,28,44]$. Several studies analyzed genome-wide gene expression changes in response to learning and have shown the requirement for $\mathrm{MeCP} 2$ for this learningdependent gene expression [21, 22, 45]. In contrast, alternative splicing changes on a genome-wide scale upon learning have been less explored. Poplawski and colleagues (2016) were the first to investigate genome-wide alternative splicing changes in the hippocampus after a contextual-fear learning and after memory recall and identified novel alternative splicing isoforms that may be critical for memory consolidation [9]. Our observations support and further expand this previous dataset by providing a novel set of alternative splicing events triggered by a non-aversive object-location learning, concluding that both aversive and non-aversive forms of learning induce genome-wide alternative splicing changes in the hippocampus.

The mechanisms through which $\mathrm{MeCP} 2$ regulate learning-dependent alternative splicing events, particularly in 


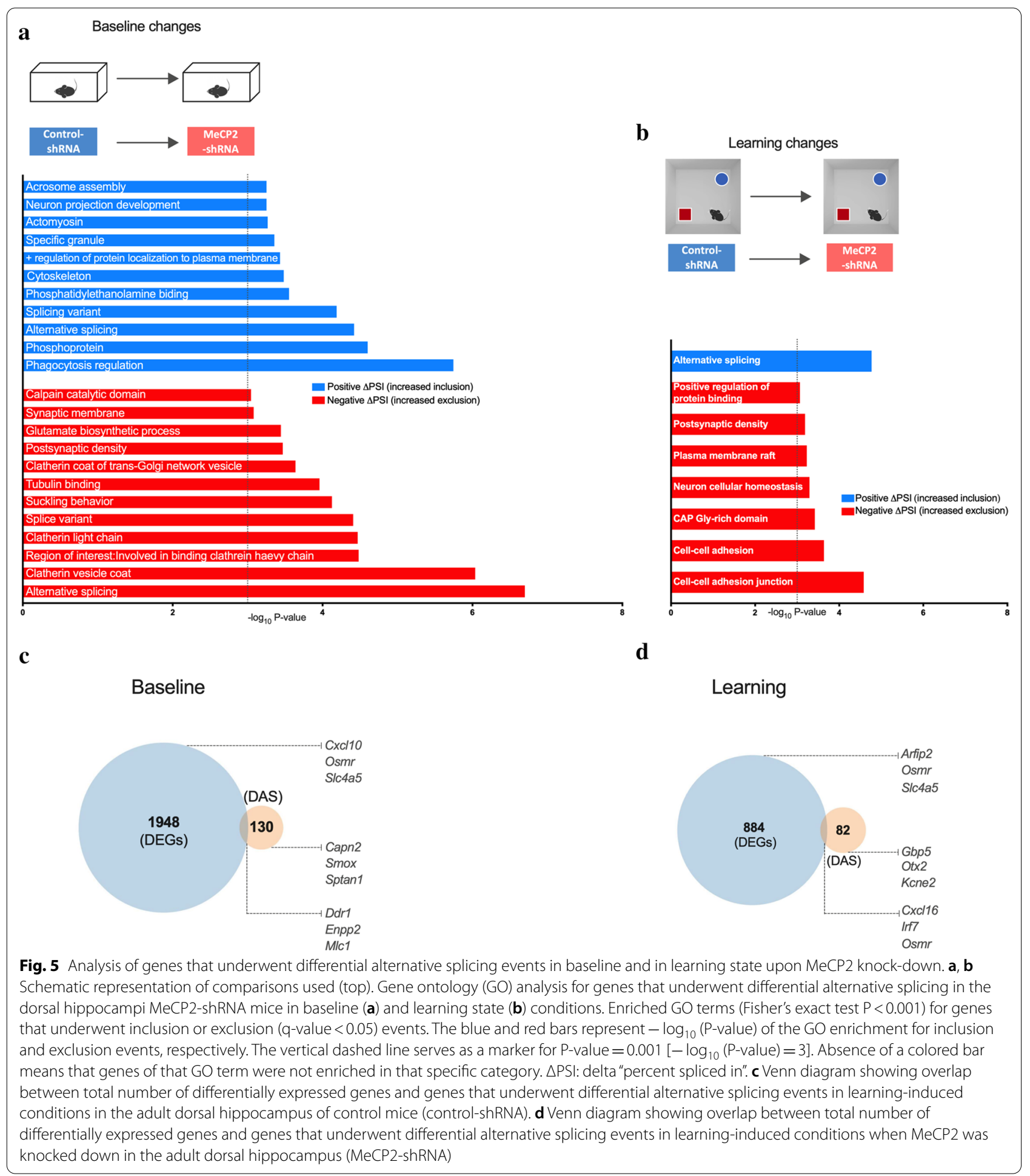

mature neurons, are poorly understood. Recently, Osenberg and colleagues [8] studied activity-dependent gene expression and alternative splicing in a mouse model of RTT. The authors elicited neuronal activity in Mecp2-null $\left(\mathrm{Mecp}^{-/ y}\right)$ mice through the administration of kainic acid and identified genome-wide alternative splicing changes in the hippocampus in response to this neuronal stimulation. They found an aberrant global pattern of gene expression and alternative splicing events. Here, we used an adult-onset knockdown of MeCP2 and induced 
neuronal activity by a physiological and memory-relevant stimulus, novel environment exposure. We found that MeCP2 knockdown led to an increase in intron retention and decreased excluded exons. Notably, Wong and colleagues (2017) showed that decreased MeCP2 binding near splice junctions facilitates intron retention via reduced recruitment of splicing factors, such as the splicing factor transformer-2 protein homolog beta (Tra2b), and stalling of RNA polymerase II [27]. In MeCP2 depletion conditions, like the one in our study, intron retention is favored possibly through the enabling of Tra2b activity. Importantly, this was not associated with an altered Tra2b expression in MeCP2-shRNA mice [21]. Moreover, intragenic DNA methylation and MeCP2 binding promote exon recognition and consequently $\mathrm{MeCP} 2$ ablation results in aberrant exon skipping events [26]. Overall, the demonstrated involvement of $\mathrm{MeCP} 2$ in these splicing modalities together with the shift towards increased retained introns and exons in MeCP2 knockdown conditions observed in our study, suggest that MeCP2 contributes to learning-induced alternative splicing through these mechanisms. Although aberrations in these splicing events were predominant, we identified learninginduced changes in other forms of alternative splicing in the hippocampus of MeCP2-shRNA mice. This indicates that $\mathrm{MeCP} 2$ may regulate other forms of splicing through mechanisms not yet identified.

In this study, we analyzed alternative splicing events in response to learning in control or MeCP2-shRNA hippocampi as well as in baseline or learning states. This combinatorial analysis allowed us to conclude that the differences found in the learning state do not only reflect changes in basal conditions, but also a requirement for $\mathrm{MeCP} 2$ in learning-dependent alternative splicing. Therefore, this indicates that the contribution of MeCP2 to synaptic plasticity and memory is likely two-fold. On the one hand, MeCP2 regulates the neuronal basal transcriptome which may impact neuronal properties such as synaptic transmission and intracellular signal transduction, and additionally may regulate directly stimulus-dependent transcriptional and post-transcriptional events in the nucleus.

$\mathrm{MeCP} 2$ is essential for the maintenance of structural and functional properties of neuronal circuits as demonstrated in RTT mouse models [20, 46-48]. We found that $\mathrm{MeCP} 2$ regulates alternative splicing of learningregulated genes relevant for synaptic plasticity. Noteworthy examples are the P2X purinoceptor $6(P 2 r \times 6)$, the neuron-glia related cell adhesion molecule (Nrcam) and the Glutamate Ionotropic Receptor AMPA Type Subunit 3 (Gria3). The P2X receptors are ligand-gated ion channels activated by extracellular ATP. Seven P2X receptor subunits have been identified (P2X1-P2X7) that form trimeric receptors of homomeric or heteromeric composition. P2X6 is present in the rodent hippocampus predominantly at glutamatergic synapses [49]. The activity of P2X receptors induces fast excitatory postsynaptic currents and has multiple modulatory effects on synaptic plasticity. In the hippocampus, P2X receptors contribute to synaptic strength modulation through its critical role in the regulation of the trafficking of AMPA receptors at the postsynaptic membrane [50-53]. Interestingly, alternative splicing regulation of $\mathrm{P} 2 \mathrm{X}$ receptors has been reported, that affects its expression pattern and possibly function $[51,54,55]$. Moreover, P2X6 subunits have been shown to translocate to the nucleus in hippocampal neurons, where they are able to interact with members of the spliceosome [50] leading to altered splicing activity. Hence, in the absence of MeCP2, the cellular function may be compromised due to a change in the relative abundance of alternatively spliced P2X6 forms and/or altered splicing activity.

Nrcam is required for cognitive function [56]. This protein is expressed at the synapse and regulates synapse formation and remodeling in the brain $[57,58]$. We found that Nrcam exon skipping was decreased in learning state in MeCP2-shRNA mice. In agreement with our findings, exon skipping of Nrcam is induced following exposure to novel objects supporting a functional role in this process [59]. These results suggest that altered splicing of Nrcam might contribute to the memory impairments observed in MeCP2-shRNA mice [21].

It is well established that glutamate receptor subunits are highly regulated by alternative splicing. RNA editing generates flip/flop variants that dynamically regulate conductance of AMPA receptors $[60,61]$. Moreover, alternative splicing of AMPA receptor subunits is induced by neuronal activity in the hippocampus [62]. We found that MeCP2-shRNA mice showed increase intron retention of the Glutamate Ionotropic Receptor AMPA Type Subunit 3 (Gria3) during learning state. Retained intron sequences present in Gria3 mRNA are responsible for its dendritic localization [63]. Importantly our findings are in agreement with a study that detected altered expression of Gria3 splicing variants in the cortex of Mecp $2 \mathrm{KO}$ mice [28] indicating that $\mathrm{MeCP} 2$ regulates Gria3 alternative splicing also in the mouse hippocampus specifically in learning conditions. Altogether this data suggests that alterations in the relative amounts of splicing isoforms of genes supporting functional and structural plasticity changes after learning may contribute to the cognitive deficits observed in MeCP2 knockdown mice [21]. It is noteworthy that acute disruptions of adult hippocampal MeCP2 did not alter the dendritic complexity and spine density of CA1 neurons in baseline conditions 
[21]. This is in line with our observations that DAS in MeCP2 knockdown in baseline conditions was not enriched for genes functionally relevant to "dendritic spine regulation". Our findings therefore suggest that $\mathrm{MeCP} 2$ regulates alternative splicing of the genes associated with dendritic spines mostly in response to learning, which may cause selective impairments in learning-dependent spine remodeling [64-66]. Whether MeCP2 disruptions alter learning-dependent structural remodeling in mature hippocampal neurons remains to be investigated.

We found that at baseline conditions MeCP2 reduction promoted an overall increase in IR and a decrease in skipped exons, particularly in genes functionally linked to general neuronal functions. Specifically, the abundance of transcript isoforms relevant for neurotransmitter synthesis (glutaminase $(G l s)$ ), vesicle recycling (synaptojanin 1 (Synj1)) and neurotransmitter receptors (gamma-aminobutyric acid (GABA) A receptor, subunit gamma 2 (Gabrg2), glutamate ionotropic receptor NMDA Type Subunit 1 (Grin1)) was altered in MeCp2 knockdown conditions. Interestingly, the Grin1 gene gives rise to 8 splice variants and recently it has been shown that the selective expression of different GluN1 isoforms determines long-term potentiation in the hippocampus and spatial memory performance [67]. Moreover, the relative abundance of some spliced isoforms of GluN1 subunit is associated with increased seizure susceptibility in adult mice [68]. Taken together, these findings suggest that altered alternative splicing events observed in MeCP2shRNA mice at baseline might impact proper neuronal function and consequently contribute to cognitive deficits and excitation/inhibition imbalance reminiscent of RTT. Furthermore, we found aberrant splicing and/or expression of splicing regulators in resting and learning conditions. In particular, MeCP2-shRNA mice during the learning state displayed changes in the abundance of U1 small nuclear ribonucleoprotein 70 (Snrnp70) and U2 small nuclear RNA auxiliary factor 1-like 4 (U2af114) spliced variants, two components of the spliceosome. In baseline conditions, MeCP2 regulates the expression of the Small the Nuclear Ribonucleoprotein U4/U6.U5 Subunit 27 (Snrnp27) and the Polypyrimidine tract-binding protein 1 (Ptbp1) [21]. These findings are in agreement with a previous study that also observed alterations in the expression and splicing of splicing regulators as a consequence of $\mathrm{MeCP} 2$ ablation [8]. It is plausible that aberrant expression and/or isoform levels of splicing mediators may induce a second wave of impairments in downstream splicing events, such as in response to learning as observed in MeCP2-shRNA mice. Furthermore, as MeCP2 interacts not only with transcription factors but also with regulators of alternative splicing [25-28, 44,
69], loss of MeCP2 may thus impair their recruitment and promote the disruption of alternative splicing events observed in MeCP2-shRNA mice.

Overall in this study, we found that spatial learning induces alternative splicing events of transcripts with relevant functions for neuronal structure and function. Moreover, our findings implicated $\mathrm{MeCP} 2$ in the regulation of this process. We showed that the reduction of MeCP2 levels in adult hippocampus promoted aberrant alternative splicing patterns in both baseline and learning states. This study uncovered another factor that likely contributes to the neuronal dysfunctions that characterize RTT.

\section{Supplementary information}

Supplementary information accompanies this paper at https://doi. org/10.1186/s13041-020-00695-1.

Additional file 1: Table S1. List of primers used for qRT-PCR and RT-PCR.

Additional file 2: Table S2. Learning-induced DAS. Description of data: This file contains the number of differential alternative splicing (DAS) events per subtype for learning-induced conditions.

Additional file 3: Figure S1. Alternative splicing event-specific changes in MeCP2 knockdown mice upon spatial learning. A) Schematic representation of the comparisons used. B-D) Pie charts showing the proportion of learning-induced inclusion and exclusion events for (B) alternative $3^{\prime}$ splice sites (A3SS), (C) alternative 5' splice (A5SS) and (D) mutually exclusive exons (ME) in Control-shRNA and MeCP2-shRNA mice. E-I) Scatter plots showing changes in (E) A3SS, (F) A5SS, (G) ME, (H) intron retention (IR) and (I) exon skipping (ES) events in Control-shRNA (Control) and MeCP2-shRNA (MeCP2KD) mice upon learning. Red dots and blue squares represent alternative splicing events occurred in either Control or MeCP2knock-down (MeCP2KD) (q-value <0.05), respectively. Green triangles represent alternative splicing events that occurred in both conditions (q-value $<0.05$ ). J-L) Violin plots showing the $\triangle P S I$ distribution of A3SS (J), A5SS (K) events in Control-shRNA and MeCP2-shRNA hippocampi after learning. The P-values are based on paired two-tailed Student's t test or Wilcoxon test and are indicated at the top of each panel. $\triangle P S I$ : delta "percent spliced in".

Additional file 4: Table S3. $\triangle \mathrm{PSI}$ for Learning-induced DAS. This file contains $\triangle P S I$ values for the differential alternative splicing (DAS) events per subtype for learning-induced conditions.

Additional file 5: Figure S2. Validation of genes that underwent differential alternative splicing. (A) Quantitative RT-PCR for differential alternative splicing events. Box plots show the relative ratio of retained vs. excised introns (IR) or included vs. excluded exons (ES, A5SS) between conditions $(\mathrm{N}=8-10)$. The diagram below each individual gene shows the two possible isoforms (included/excluded exon or intron in red or blue, respectively) with arrows indicating the location of the primers used (Additional file 1: Table S1). The P-values are based on unpaired two-tailed Student's t test or Mann-Whitney test indicated at the top of each panel. (B) Semiquantitative RT-PCR for genes that underwent differential alternative splicing. Gel images show the retained vs. spliced isoform intensity. Diagram next to gene name shows the location of the primers used (Additional file 1: Table S1). Three biological replicates are shown per condition. *refers to unspecific PCR products based on size prediction.

Additional file 6: Table S4. GO analysis and DEG/DAS overlap for Learning-induced conditions. This file contains gene ontology (GO) analysis and overlap for DEGs and DAS for learning-induced conditions.

Additional file 7: Table S5. Baseline and learning state DAS. This file contains the number of differential alternative splicing (DAS) events per subtype for baseline and learning state conditions. 
Additional file 8: Table S6. $\triangle \mathrm{PSI}$ for Baseline and learning state DAS. This file contains $\triangle \mathrm{PSI}$ values from the differential alternative splicing (DAS) events per subtype for baseline and learning-state conditions.

Additional file 9: Figure S3. Alternative splicing event-specific changes in MeCP2 knock-down mice during baseline and learning state conditions. A) Schematic representation of the comparisons used. B-D) Pie charts showing the proportion of inclusion and exclusion events for (B) alternative $3^{\prime}$ splice sites (A3SS), and (C) alternative $5^{\prime}$ splice (A5SS) and (D) mutually exclusive exons (ME) in MeCP2-shRNA mice during baseline and learning-state conditions. Note that no ME changes were detected by MeCP2 knockdown in learning-state. E-I) Scatter plots showing changes in (E) A3SS, (F) A5SS, (G) ME, (H) intron retention (IR) and (I) exon skipping (ES) events in home-cage (Baseline) and learning state (Learning) conditions in MeCP2-shRNA mice compared to the controls. Red dots and blue squares represent alternative splicing events occurred in either baseline or learning conditions (q-value $<0.05$ ), respectively. Green triangles represent alternative splicing events that occurred in both conditions (q-value $<0.05$ ). J-L) Violin plots showing the $\triangle P S I$ distribution of (J) A3SS, (K) A5SS and (L) ME events in baseline and learning state conditions in the dorsal hippocampi of MeCP2-shRNA mice. The P-values are based on paired two-tailed Student's t test or Wilcoxon test. and are indicated at the top of each panel. $\triangle$ PSI: delta "percent spliced in".

Additional file 10: Table S7. GO analysis and DEG/DAS overlap for baseline and learning state conditions. This file contains gene ontology (GO) analysis and overlap for DEGs and DAS for baseline and learning conditions.

\section{Abbreviations}

Atl2: Atlastin GTPase 2; A3SS: Alternative 3' splice sites; A5SS: Alternative 5' splice sites; BLAST: Basic Local Alignment Search Tool; CamKlla: Calcium/ calmodulin-dependent protein kinase type II subunit alpha; cDNA: Complementary deoxyribonucleic acid; DAS: Alternative splicing events; DAVID: Database for annotation, visualization and integrated discovery; Dnajb5: DnaJ Heat Shock Protein Family (Hsp40) Member B5; DEG: Differentially expressed gene; ES: Exon skipping; FDR: False discovery rate; Fhl1: Four And A Half LIM Domains 1; Gabrg2: Gamma-aminobutyric acid (GABA) A receptor, subunit gamma 2; GEO: Gene expression omnibus; Gls: Glutaminase; GO: Gene ontology; Gria3: Glutamate ionotropic receptor AMPA type subunit 3; Irf7: Interferon regulatory factor 7; IR: Intron retention; LuC7|3: LUC7 like 3 pre-MRNA splicing factor; Map4: Microtubule-associated protein 4; Marchf7: Membrane associated ring-CH-type finger 7; MATS: Multivariate analysis of transcript splicing; ME: Mutually exclusive exons; MeCP2: Methyl CpG binding domain protein 2; NCBI: National Center for Biotechnology Information; Nr3c1: Nuclear receptor subfamily 3, group C, member 1; Nrcam: Neuron-glia related cell adhesion molecule; Osmr: Oncostatin M receptor; P2rx6: P2X purinoceptor 6; PCR: Polymerase chain reaction; qRT-PCR: Real-time quantitative reverse transcription PCR; rAAV: Recombinant adeno-associated virus; RNA: Ribonucleic acid; RNAseq: RNA sequencing; RT-PCR: Reverse transcription polymerase chain reaction; RTT: Rett Syndrome; shRNA: Short-hairpin RNA; Snrnp70: U1 small nuclear ribonucleoprotein 70; Synj1: Synaptojanin 1; Tra2b: Transformer-2 protein homolog beta; Trmt1: TRNA (guanine(26)-N(2))-dimethyltransferase; U2af1/4: U2 small nuclear RNA auxiliary factor 1-like 4; YB-1: Y-box binding protein 1; Zmynd8: Zinc finger, MYND-type containing 8; $\triangle \mathrm{PSI}$ : Percent spliced in.

\section{Acknowledgements}

We thank to Benjamin Zeuch for producing the viruses and providing technical assistance throughout the project.

\section{Authors' contributions}

D.V.C.B., K.G.K. and A.M.M.O. conceived the project and designed research; D.V.C.B., K.G.K., J.K. and L.F. performed research; D.V.C.B. and J.K. analyzed data; D.V.C.B., K.G.K. and A.M.M.O. wrote the manuscript. All authors read and approved the final manuscript.

\section{Funding}

Open Access funding enabled and organized by Projekt DEAL. This work was supported by the Deutsche Forschungsgemeinschaft (DFG) [SFB 1134 (project C01), an Emmy Noether grant (OL 437/1) and research grant (OL 437/2) to
A.M.M.O.] and by the Chica and Heinz Schaller foundation [fellowship and research award to A.M.M.O.]. D.V.C.B. is supported by a Landesgraduiertenförderung (LGF) completion grant (Heidelberg Graduate Academy).

\section{Availability of data and materials}

The datasets generated and analyzed during the current study are available at the National Center for Biotechnology Information (NCBI) Gene Expression Omnibus (GEO) with the accession number GSE107004 and are included in the additional files.

\section{Ethics approval and consent to participate}

All animal experiments were done in accordance with German guidelines for the care and use of laboratory animals and with the European Community Council Directive 2010/63/EU. Experiments were approved by local authorities (Regierungspräsidium Karlsruhe, Germany).

\section{Consent for publication}

Not applicable.

\section{Competing interests}

The authors declare that they have no competing interests.

\section{Author details}

${ }^{1}$ Department of Neurobiology, Interdisciplinary Centre for Neurosciences (IZN), Heidelberg University, Im Neuenheimer Feld 366, 69120 Heidelberg, Germany. ${ }^{2}$ Division of Chromatin Networks, German Cancer Research Center (DKFZ) and Bioquant (Heidelberg University), Heidelberg, Germany. ${ }^{3}$ Present Address: Department of Cognitive Neuroscience, Radboud University Medical Center, 6500 HB Nijmegen, The Netherlands. ${ }^{4}$ Present Address: Donders Institute for Brain, Cognition and Behaviour, Radboud University Nijmegen, 6525 EN Nijmegen, The Netherlands.

Received: 18 June 2020 Accepted: 6 November 2020

Published online: 17 November 2020

\section{References}

1. Yap EL, Greenberg ME. Activity-regulated transcription: bridging the gap between neural activity and behavior. Neuron. 2018;100:330-48.

2. Hernandez PJ, Abel T. The role of protein synthesis in memory consolidation: progress amid decades of debate. Neurobiol Learn Mem. 2008;89:293-311.

3. Brito DVC, Gulmez Karaca K. Neuronal chromatin architecture regulator CTCF dictates remote memory. J Neurosci. 2018;38:10239-40.

4. Rajarajan P, Gil SE, Brennand KJ, Akbarian S. Spatial genome organization and cognition. Nat Rev Neurosci. 2016;17:681-91.

5. Oliveira AM. DNA methylation: a permissive mark in memory formation and maintenance. Learn Mem. 2016;23:587-93.

6. Beagan JA, Pastuzyn ED, Fernandez LR, Guo MH, Feng K, Titus KR, Chandrashekar H, Shepherd JD, Phillips-Cremins JE. Three-dimensional genome restructuring across timescales of activity-induced neuronal gene expression. Nat Neurosci. 2020;23:707-17.

7. Su Y, Shin J, Zhong C, Wang S, Roychowdhury P, Lim J, Kim D, Ming GL, Song H. Neuronal activity modifies the chromatin accessibility landscape in the adult brain. Nat Neurosci. 2017;20:476-83.

8. Osenberg S, Karten A, Sun J, Li J, Charkowick S, Felice CA, Kritzer M, Nguyen MVC, Yu P, Ballas N. Activity-dependent aberrations in gene expression and alternative splicing in a mouse model of Rett syndrome. Proc Natl Acad Sci U S A. 2018;115:E5363-e5372.

9. Poplawski SG, Peixoto L, Porcari GS, Wimmer ME, McNally AG, Mizuno K, Giese KP, Chatterjee S, Koberstein JN, Risso D, et al. Contextual fear conditioning induces differential alternative splicing. Neurobiol Learn Mem. 2016;134(Pt B):221-35.

10. Mauger $\mathrm{O}$, Lemoine F, Scheiffele P. Targeted intron retention and excision for rapid gene regulation in response to neuronal activity. Neuron. 2016;92:1266-78. 
11. lijima T, Wu K, Witte H, Hanno-lijima Y, Glatter T, Richard S, Scheiffele P. SAM68 regulates neuronal activity-dependent alternative splicing of neurexin-1. Cell. 2011;147:1601-14.

12. An P, Grabowski PJ. Exon silencing by UAGG motifs in response to neuronal excitation. PLoS Biol. 2007:5:e36.

13. Porter RS, Jaamour F, Iwase S. Neuron-specific alternative splicing of transcriptional machineries: implications for neurodevelopmental disorders. Mol Cell Neurosci. 2018;87:35-45.

14. Vuong CK, Black DL, Zheng S. The neurogenetics of alternative splicing. Nat Rev Neurosci. 2016;17:265-81.

15. Ellis JD, Barrios-Rodiles M, Colak R, Irimia M, Kim T, Calarco JA, Wang X, Pan Q, O'Hanlon D, Kim PM, et al. Tissue-specific alternative splicing remodels protein-protein interaction networks. Mol Cell. 2012;46:884-92.

16. Braunschweig U, Barbosa-Morais NL, Pan Q, Nachman EN, Alipanahi B, Gonatopoulos-Pournatzis T, Frey B, Irimia M, Blencowe BJ. Widespread intron retention in mammals functionally tunes transcriptomes. Genome Res. 2014;24:1774-86.

17. Baker-Andresen D, Ratnu VS, Bredy TW. Dynamic DNA methylation: a prime candidate for genomic metaplasticity and behavioral adaptation. Trends Neurosci. 2013:36:3-13.

18. Gulmez Karaca K, Brito DVC, Oliveira AMM. MeCP2: a critical regulator of chromatin in neurodevelopment and adult brain function. Int J Mol Sci. 2019;20:4577.

19. Guy J, Cheval H, Selfridge J, Bird A. The role of MeCP2 in the brain. Annu Rev Cell Dev Biol. 2011;27:631-52.

20. Ip JPK, Mellios N, Sur M. Rett syndrome: insights into genetic, molecular and circuit mechanisms. Nat Rev Neurosci. 2018;19:368-82.

21. Gulmez Karaca K, Brito DVC, Zeuch B, Oliveira AMM. Adult hippocampal MeCP2 preserves the genomic responsiveness to learning required for long-term memory formation. Neurobiol Learn Mem. 2018;149:84-97.

22. Chahrour M, Jung SY, Shaw C, Zhou X, Wong ST, Qin J, Zoghbi HY. MeCP2, a key contributor to neurological disease, activates and represses transcription. Science. 2008:320:1224-9.

23. Sanfeliu A, Kaufmann WE, Gill M, Guasoni P, Tropea D. Transcriptomic studies in mouse models of Rett syndrome: a review. Neuroscience. 2019;413:183-205.

24. Sanfeliu A, Hokamp K, Gill M, Tropea D. Transcriptomic analysis of Mecp2 mutant mice reveals differentially expressed genes and altered mechanisms in both blood and brain. Front Psychiatry. 2019;10:278.

25. Young Jl, Hong EP, Castle JC, Crespo-Barreto J, Bowman AB, Rose MF, Kang D, Richman R, Johnson JM, Berget S, Zoghbi HY. Regulation of RNA splicing by the methylation-dependent transcriptional repressor methylCpG binding protein 2. Proc Natl Acad Sci U S A. 2005;102:17551-8.

26. Maunakea AK, Chepelev I, Cui K, Zhao K. Intragenic DNA methylation modulates alternative splicing by recruiting MeCP2 to promote exon recognition. Cell Res. 2013;23:1256-69.

27. Wong JJ, Gao D, Nguyen TV, Kwok CT, van Geldermalsen M, Middleton R, Pinello N, Thoeng A, Nagarajah R, Holst J, et al. Intron retention is regulated by altered $\mathrm{MeCP} 2$-mediated splicing factor recruitment. Nat Commun. 2017;8:15134

28. Li R, Dong Q, Yuan X, Zeng X, Gao Y, Chiao C, Li H, Zhao X, Keles S, Wang Z, Chang Q. Misregulation of alternative splicing in a mouse model of Rett syndrome. PLoS Genet. 2016;12:e1006129.

29. Gulmez Karaca K, Kupke J, Brito DVC, Zeuch B, Thome C, Weichenhan D, Lutsik P, Plass C, Oliveira AMM. Neuronal ensemble-specific DNA methylation strengthens engram stability. Nat Commun. 2020;11:639.

30. Brito DVC, Kupke J, Gulmez Karaca K, Zeuch B, Oliveira AMM. Mimicking age-associated Gadd45gamma dysregulation results in memory impairments in young adult mice. J Neurosci. 2020;40:1197-210.

31. Oliveira AM, Hemstedt TJ, Bading H. Rescue of aging-associated decline in Dnmt3a2 expression restores cognitive abilities. Nat Neurosci. 2012;15:1111-3.

32. Shen S, Park JW, Huang J, Dittmar KA, Lu ZX, Zhou Q, Carstens RP, Xing Y. MATS: a Bayesian framework for flexible detection of differential alternative splicing from RNA-Seq data. Nucleic Acids Res. 2012;40:e61.

33. Langmead B, Trapnell C, Pop M, Salzberg SL. Ultrafast and memoryefficient alignment of short DNA sequences to the human genome. Genome Biol. 2009;10:R25.

34. Trapnell C, Roberts A, Goff L, Pertea G, Kim D, Kelley DR, Pimentel H, Salzberg SL, Rinn JL, Pachter L. Differential gene and transcript expression analysis of RNA-seq experiments with TopHat and Cufflinks. Nat Protoc. 2012;7:562-78.

35. Burger C, Gorbatyuk OS, Velardo MJ, Peden CS, Williams P, Zolotukhin S, Reier PJ, Mandel RJ, Muzyczka N. Recombinant AAV viral vectors pseudotyped with viral capsids from serotypes 1, 2, and 5 display differential efficiency and cell tropism after delivery to different regions of the central nervous system. Mol Ther. 2004;10:302-17.

36. Xu R, Janson CG, Mastakov M, Lawlor P, Young D, Mouravlev A, Fitzsimons $\mathrm{H}$, Choi KL, Ma H, Dragunow $\mathrm{M}$, et al. Quantitative comparison of expression with adeno-associated virus (AAV-2) brain-specific gene cassettes. Gene Ther. 2001;8:1323-32.

37. Amir RE, Van den Veyver IB, Wan M, Tran CQ, Francke U, Zoghbi HY. Rett syndrome is caused by mutations in X-linked MECP2, encoding methyl-CpG-binding protein 2. Nat Genet. 1999;23:185-8.

38. Cheval H, Guy J, Merusi C, De Sousa D, Selfridge J, Bird A. Postnatal inactivation reveals enhanced requirement for MeCP2 at distinct age windows. Hum Mol Genet. 2012;21:3806-14.

39. Gemelli T, Berton O, Nelson ED, Perrotti LI, Jaenisch R, Monteggia LM. Postnatal loss of methyl-CpG binding protein 2 in the forebrain is sufficient to mediate behavioral aspects of Rett syndrome in mice. Biol Psychiatry. 2006;59:468-76.

40. McGraw CM, Samaco RC, Zoghbi HY. Adult neural function requires MeCP2. Science. 2011;333:186.

41. Moretti P, Levenson JM, Battaglia F, Atkinson R, Teague R, Antalffy B, Armstrong D, Arancio O, Sweatt JD, Zoghbi HY. Learning and memory and synaptic plasticity are impaired in a mouse model of Rett syndrome. J Neurosci. 2006;26:319-27.

42. Nguyen MV, Du F, Felice CA, Shan X, Nigam A, Mandel G, Robinson JK, Ballas N. MeCP2 is critical for maintaining mature neuronal networks and global brain anatomy during late stages of postnatal brain development and in the mature adult brain. J Neurosci. 2012;32:10021-34.

43. Skene PJ, Illingworth RS, Webb S, Kerr AR, James KD, Turner DJ, Andrews R, Bird AP. Neuronal MeCP2 is expressed at near histoneoctamer levels and globally alters the chromatin state. Mol Cell. 2010;37:457-68.

44. Cheng J, Huang M, Zhu Y, Xin YJ, Zhao YK, Huang J, Yu JX, Zhou WH, Qiu Z. SUMOylation of MeCP2 is essential for transcriptional repression and hippocampal synapse development. J Neurochem. 2014;128:798-806.

45. Deng JV, Rodriguiz RM, Hutchinson AN, Kim IH, Wetsel WC, West AE. $\mathrm{MeCP} 2$ in the nucleus accumbens contributes to neural and behavioral responses to psychostimulants. Nat Neurosci. 2010;13:1128-36.

46. Chapleau CA, Calfa GD, Lane MC, Albertson AJ, Larimore JL, Kudo S, Armstrong DL, Percy AK, Pozzo-Miller L. Dendritic spine pathologies in hippocampal pyramidal neurons from Rett syndrome brain and after expression of Rett-associated MECP2 mutations. Neurobiol Dis. 2009;35:219-33.

47. Kishi N, Macklis JD. MECP2 is progressively expressed in post-migratory neurons and is involved in neuronal maturation rather than cell fate decisions. Mol Cell Neurosci. 2004;27:306-21.

48. Na ES, Nelson ED, Kavalali ET, Monteggia LM. The impact of MeCP2 loss- or gain-of-function on synaptic plasticity. Neuropsychopharmacology. 2013;38:212-9.

49. Rubio ME, Soto F. Distinct Localization of $P 2 X$ receptors at excitatory postsynaptic specializations. J Neurosci. 2001;21:641-53.

50. Diaz-Hernandez Jl, Sebastian-Serrano A, Gomez-Villafuertes R, DiazHernandez M, Miras-Portugal MT. Age-related nuclear translocation of P2X6 subunit modifies splicing activity interacting with splicing factor 3A1. PLOS ONE. 2015;10:e0123121.

51. Kaczmarek-Hajek K, Lorinczi E, Hausmann R, Nicke A. Molecular and functional properties of $\mathrm{P} 2 \mathrm{X}$ receptors - recent progress and persisting challenges. Purinergic Signal. 2012;8:375-417.

52. Pougnet JT, Compans B, Martinez A, Choquet D, Hosy E, Boué-Grabot E. $\mathrm{P} 2 \mathrm{X}$-mediated AMPA receptor internalization and synaptic depression is controlled by two CaMKII phosphorylation sites on GluA1 in hippocampal neurons. Sci Rep. 2016;6:31836.

53. Pougnet JT, Toulme E, Martinez A, Choquet D, Hosy E, Boue-Grabot E. ATP P2X receptors downregulate AMPA receptor trafficking and postsynaptic efficacy in hippocampal neurons. Neuron. 2014;83:417-30.

54. da Silva RL, Resende RR, Ulrich $H$. Alternative splicing of $P 2 X 6$ receptors in developing mouse brain and during in vitro neuronal differentiation. Exp Physiol. 2007;92:139-45. 
55. Masin M, Kerschensteiner D, Dumke K, Rubio ME, Soto F. Fe65 interacts with P2X2 subunits at excitatory synapses and modulates receptor function. J Biol Chem. 2006;281:4100-8.

56. Moy SS, Nonneman RJ, Young NB, Demyanenko GP, Maness PF. Impaired sociability and cognitive function in Nrcam-null mice. Behav Brain Res. 2009;205:123-31.

57. Demyanenko GP, Mohan V, Zhang X, Brennaman LH, Dharbal KE, Tran TS, Manis PB, Maness PF. Neural cell adhesion molecule NrCAM regulates Semaphorin 3F-induced dendritic spine remodeling. J Neurosci. 2014;34:11274-87.

58. Sakurai T. The role of NrCAM in neural development and disordersbeyond a simple glue in the brain. Mol Cell Neurosci. 2012;49:351-63.

59. Scott H, Rogers MF, Scott HL, Campbell C, Warburton EC, Uney JB. Recognition memory-induced gene expression in the perirhinal cortex: a transcriptomic analysis. Behav Brain Res. 2017;328:1-12.

60. La Via L, Bonini D, Russo I, Orlandi C, Barlati S, Barbon A. Modulation of dendritic AMPA receptor mRNA trafficking by RNA splicing and editing. Nucleic Acids Res. 2013:41:617-31.

61. Sommer B, Keinanen K, Verdoorn TA, Wisden W, Burnashev N, Herb A, Kohler M, Takagi T, Sakmann B, Seeburg PH. Flip and flop: a cell-specific functional switch in glutamate-operated channels of the CNS. Science. 1990;249:1580-5.

62. Balik A, Penn AC, Nemoda Z, Greger IH. Activity-regulated RNA editing in select neuronal subfields in hippocampus. Nucleic Acids Res. 2013:41:1124-34.

63. Buckley PT, Lee MT, Sul JY, Miyashiro KY, Bell TJ, Fisher SA, Kim J, Eberwine J. Cytoplasmic intron sequence-retaining transcripts can be dendritically targeted via ID element retrotransposons. Neuron. 2011;69:877-84.
64. Attardo A, Fitzgerald JE, Schnitzer MJ. Impermanence of dendritic spines in live adult CA1 hippocampus. Nature. 2015;523:592-6.

65. Moser MB, Trommald M, Andersen P. An increase in dendritic spine density on hippocampal CA1 pyramidal cells following spatial learning in adult rats suggests the formation of new synapses. Proc Natl Acad Sci U S A. 1994;91:12673-5

66. Sanders J, Cowansage K, Baumgartel K, Mayford M. Elimination of dendritic spines with long-term memory is specific to active circuits. $J$ Neurosci. 2012:32:12570-8.

67. Sengar AS, Li H, Zhang W, Leung C, Ramani AK, Saw NM, Wang Y, Tu Y, Ross PJ, Scherer SW, et al. Control of long-term synaptic potentiation and learning by alternative splicing of the NMDA receptor subunit GluN1. Cell Rep. 2019;29(4285-4294):e4285.

68. Liu H, Wang H, Peterson M, Zhang W, Hou G, Zhang ZW. N-terminal alternative splicing of GluN1 regulates the maturation of excitatory synapses and seizure susceptibility. Proc Natl Acad Sci U S A. 2019;116:21207-12.

69. Lev Maor G, Yearim A, Ast G. The alternative role of DNA methylation in splicing regulation. Trends Genet. 2015;31:274-80.

\section{Publisher's Note}

Springer Nature remains neutral with regard to jurisdictional claims in published maps and institutional affiliations.
Ready to submit your research? Choose BMC and benefit from:

- fast, convenient online submission

- thorough peer review by experienced researchers in your field

- rapid publication on acceptance

- support for research data, including large and complex data types

- gold Open Access which fosters wider collaboration and increased citations

- maximum visibility for your research: over $100 \mathrm{M}$ website views per year

At BMC, research is always in progress.

Learn more biomedcentral.com/submissions 